\title{
Design and CFD Analysis of a Radial-Inflow Turbine for Small Scale ORC Applications
}

\author{
Alessandro Cappiello ${ }^{1, *}$, and Raffaele Tuccillo ${ }^{1}$ \\ ${ }^{1}$ Università di Napoli Federico II, Industrial Engineering Department, 80125 Naples, Italy
}

\begin{abstract}
In recent years, Organic Rankine Cycle (ORC) technology has received growing interests, thanks to its high flexibility and to the capability to exploit energy sources at temperature levels difficult to be approached with conventional power cycles. These features allow exploiting renewable and renewable-equivalent energy sources, by either improving the energy conversion efficiency of existing plants or using waste heat from industrial process. As far as the expander is concerned, a high potential solution is represented by turbo-expanders, which allow reduction of plant clutter and complexity, so enhancing the potential impact on the diffusion of small power ORC-based plants. The present work concerns the design of a RadialInflow Turbine for a bottoming Organic Rankine Cycle in the tens of kW scale. Design boundary conditions are retrieved by a zero-dimensional model of a solar-assisted micro gas turbine in cogenerating mode. The design process is started by means of an in-house mean-line design code accounting for real gas properties. The code is used to carry out parametric analyses to investigate the design space for several working fluids encompassing different classes, namely refrigerants and siloxanes. The program is used to assess the effect of design variables and working fluid on the turbine performance and turbine design characteristics. Subsequently, the most promising design candidates are selected and three-dimensional first guess stator and rotor geometries are built on these preliminary designs. Stationary and rotating passages are then meshed and analyzed by means of RANS CFD based solution of the stator - rotor interaction.
\end{abstract}

\section{Introduction}

More and more stringent emission regulations along with increasing attention to environmental issues have led the Organic Rankine Cycle (ORC) technology to a growing interest both from scientific community and market, as witnessed by the continuous growth of the installed ORC-based units, [1]. This can be ascribed to the high flexibility offered by this technology, which features the capability to exploit heat sources at temperature levels difficulty approachable by conventional power cycles.

The most critical component from cycle efficiency point of view is the expander [2]. In this regard, turbo-expanders feature a high potential particularly for small power application

\footnotetext{
*Corresponding author: alessandro.cappiello@unina.it
} 
e.g. waste heat recovery from automotive engines, thanks to more favorable compactness, lightweight and power density than their volumetric counterpart [3].

Organic fluids often exhibit large molecular mass, leading to low sound speed values. Additionally, organic mediums are commonly characterized by large molecular complexity, largely affecting the shape of the saturation curve, leading to wet, isentropic and dry fluids according to the saturated vapor curve slope, as well as changing the heat share between liquid preheating and fluid vaporization [4]. Furthermore, for a given expansion ratio, the enthalpy drop of organic medium is comparatively lower than steam, so allowing the use of single stage machines. On the other hand, the large expansion ratios that result from the matching with conventional heat sources, in combination with low sound speeds, usually lead to transonic and supersonic flows within the turbine passages, making the design of a single stage machine challenging. As a consequence, conventional design guidelines developed for ideal gases, might not generally hold for ORC turbines. In this regard, Mounier et al. [5] provided an update of Baljè's diagram to small radial machines operating with organic fluids at high expansion ratios and they found that the shape of the iso-efficiency curves was altered. $\mathrm{Da}$ Lio et al. [6] by means of the preliminary design method by Aungier [7,8] showed that, for a RIT expanding R245fa, the optimal specific speed was lower than that achieved with air ideal gas and that optimal velocity ratio was departing from the air ideal gas one at high volumetric expansion ratio only. Additionally, they found that optimal volumetric expansion ratio was larger for radial-inflow turbine than for axial-flow type, together with slightly larger efficiency values. Similar conclusions were also drawn by Bahamonde et al. [9] which performed a combined thermodynamic cycle-turbine preliminary design optimization, for three turbine configurations, namely axial-flow, radial-inflow and radial-outflow turbines. Perdichizzi and Lozza [10] investigated the effects of design variables for optimal and suboptimal Ns by means of a mean-line model and proposed the introduction of size parameter and volumetric expansion ratio to extend the validity of the results to other fluids. Fiaschi et al. [11] presented a mean-line model for the design of hybrid solar-geothermal low temperature application. De Servi et al. [12] presented a design process of high-speed hightemperature application RIT starting from the meanline design up to the CFD-based turbine shape optimization.

The current work presents the preliminary design and the CFD analysis of ORC RadialInflow Turbines (RIT) for a bottoming power plant considering two different working fluids.

The boundary conditions for the turbine design are obtained by a zero-dimensional model of a solar assisted micro gas turbine in cogenerating mode, the heat source for the ORC unit being the residual energy in the exhausts.

The preliminary design is accomplished by means of an in-house code that performs the sizing of inlet volute, vaned stator, vaneless interspace and rotor; thermodynamic properties and losses are computed by means of thermophysical properties library and suitable loss models, respectively. The preliminary design is carried out by varying design variables, so to investigate the design space and assessing design variables influence on turbine performance. Once suitable preliminary designs are selected for each fluid, three-dimensional vane and blade shapes are built based on the preliminary design specifications and the vane and blade passages are further analyzed by means of steady-state RANS CFD calculations.

\section{Methodology}

\subsection{Loss Correlations}

In order to accomplish the preliminary design of turbomachines, suitable loss models are required to estimate loss contributions and the choice of the loss model is of paramount 
importance for the reliability of the preliminary design. Klonowicz et al. [13], considering an AXT turbine, showed that the predicted efficiency can vary up to $15 \%$ depending on the loss model and that in some cases trends can be altered as well.

Several authors tried to extend the validity of existing correlations developed for ideal gas to organic medium either by combining several loss models [14,15] or by means of the introduction of calibration coefficients and optimization strategies [16]. In the present work it was decided to use the correlation set provided by Meroni et al. [16], as it was deemed the most appropriate thanks to the applicability to on- and off-design points and high expansion ratio turbines.

Table 1. Turbine Design Variables List

\begin{tabular}{lcl}
\hline Design Variable & Symbol/Definition & Range \\
\hline Pressure-Based Degree of Reaction & $r_{p}=\left(p_{4}-p_{5}\right) /\left(p_{01}-p_{5}\right)$ & $0.025-0.6$ \\
Radius Ratio & $\chi=R_{5 m} / R_{4}$ & $0.3-0.7$ \\
Meridional Velocity Ratio & $\mu=c_{5 m} / c_{4 m}$ & $1.0-1.4$ \\
Specific Speed & $N_{S}=\omega \sqrt{\dot{V}_{5, i s}} /\left(H_{01}-h_{5, i s}\right)^{3 / 4}$ & $0.1-1.0$ \\
Deviation from Optimal Kinematic ratio & $\Delta$ & 0 \\
Stator Inlet Flow Angle & $\alpha_{2}$ & 45 \\
Rotor Inlet Flow Angle & $\alpha_{4}$ & 75 \\
Nozzle Solidity & $\sigma_{N}$ & 1.20 \\
Stator Radius Ratio & $R_{2} / R_{3}$ & 1.10 \\
Vaneless Interspace Radius Ratio & $R_{3} / R_{4}$ & 1.02 \\
\hline
\end{tabular}

\subsection{Preliminary Design}

The preliminary design is accomplished by means of an in-house code implemented in Matlab environment, which performs the preliminary sizing of volute (treated as isentropic), stator, vaneless interspace and rotor, whose global structure is similar to the code presented by the authors in Ref. [17] for preliminary design of an axial-flow turbine. However, some additional design variables were introduced to account for the change in turbine layout, as well as some others were replaced. In Tab. 1 a complete list of the required design variables is provided. It is worth mentioning that in spite of the assumption of optimal kinematic ratio, i.e. $\left(U_{4} / c_{4}\right)_{o p t}$, a deviation from the optimal value, expressed as percentage, was introduced as design variable to account for the fact that minimum overall losses do not necessarily occur at minimum leaving kinetic energy. Furthermore, the code was linked to CoolProp thermodynamic library for fluid properties evaluation [18].

Once the program has read the boundary conditions and decision variables from input files, it performs the stage isentropic sizing, which allows the static pressure values to be set at each station from stator inlet to rotor outlet. Based on first guess loss coefficients values, the non-isentropic stage sizing is performed, retrieving stator and rotor flow and metal angles, blade and vane numbers, radius and passages height, as well as thermo-fluid-dynamic properties at each station, say temperature, pressure, density, velocity, Mach numbers. Subsequently, losses are computed via loss correlations from Ref. [16], which allows a convergence check on the three enthalpy loss coefficients, i.e. stator, vaneless interspace and rotor. If convergence criterion is not met, the program iterates establishing an iterative procedure looping between stage sizing and loss evaluation up to convergence on enthalpy loss coefficients. Furthermore, it is worth mentioning that, concerning rotor outlet station, midspan properties are first determined. Subsequently, hub and shroud properties, together 
with blade metal angles, are found by means of Isentropic Simple Radial Equilibrium, by imposing a free-vortex distribution.

\subsection{Three-Dimensional Geometry}

Once suitable turbine candidates were found, a 3D geometry was built for both stator and rotor. Depending on the stator outlet Mach number value found in the preliminary design, purely convergent or convergent-divergent stators might be required. In the former case, the a radial-inflow nozzle is built directly in ANSYS BladeGen, by providing the main geometrical features found in the preliminary design, say leading edge and trailing edge thicknesses, vane number, inlet and outlet metal angles, vane chord and stagger angle.

For higher stator discharge Mach number, convergent-divergent vanes are necessary, and several steps are required to build the final nozzle shape. Some methodologies based on an adapted Method of Characteristics (MoC) for the design of convergent-divergent radialinflow nozzles have been presented over the years, say Ref. [19, 20, 21].

In the present work, the divergent part of a two-dimensional sharp-edged throat minimum length convergent-divergent nozzle is designed by means of the Method of Characteristics (MoC), Ref. [22], adapted to dense gases, Ref. [23], assuming straight sonic line. Subsequently, the nozzle size is scaled to deliver the design mass flow rate divided by the required vane number.

The divergent part of the nozzle is then arranged into a stator row by rotating it to the desired outlet angle and the supersonic part of the pressure-side is achieved by rotating the lower nozzle contour of an angular pitch. A small circular arc is placed on the pressure-side, before the sharp-edged throat, to promote flow acceleration, compensating the characteristic S-shaped sonic line. Second-order Bezier polynomials are used to build the uncovered part of the suction side, as well as the subsonic part of the pressure side. Finally, the nose of the vane is made by means of two circular arcs of different radii: a larger one in the proximity of the throat, where gradients are higher, and a smaller one for the Leading Edge (LE). Points coordinates defining hub, shroud and blade profile are then transferred to ANSYS TurboGrid for meshing.

The rotor was built by means of Aungier's first guess geometry method [8], which is briefly described in the forthcoming.

\subsubsection{Meridional channel}

The hub contour is constructed by means of $90^{\circ}$ circular arc whose radius of curvature is found as Eq. 1, with rotor axial length being determined by Eq. 2; the remaining part of the hub contour is a straight line located either at the outlet of the meridional channel, first case Eq. 1, or at the inlet, second case in Eq. 1.

Shroud contour is constructed by means of a power law, Eq. 3 and 4, with an exponent $n$, between 2 and 9: Aungier recommends the values which produces an area value at half of the meridional coordinate closer to arithmetic average of inlet and outlet areas, Ref. [8].

\subsubsection{Blade camber line and thickness distribution}

ORC turbines feature lower rotational speed than ideal gases applications, say turbocharger, alleviating the blade structural integrity concerns. In view of the above consideration, it was chosen not to use radial-fibered blades (which relieves structural load but on the other hand imposes additional constraints on the blade angles change along the blade span), to enhance the degrees of freedom of the design. Hence, hub and shroud camber lines were determined via polynomial laws for blade wrap angles, Eq. 5. Moreover, for their computation, inlet and 
outlet wrap angles were set to values equivalent to the hub and shroud blade metal angles (from the preliminary design), respectively, Eq. 6 and 7. It is worth mentioning that, as a consequence of the free-vortex distribution for tangential velocity component, the outlet metal angle varies from hub to shroud, whereas rotor inlet metal angle is assumed constant from hub to shroud.

The blade thickness distribution is assumed to be constant for the first $90 \%$ of the meridional coordinate and equal to leading edge thickness value; for the remaining $10 \%$ a linear distribution from the leading edge thickness to the trailing edge thickness is imposed. The above-mentioned set of information is fed to ANSYS BladeGen via a Matlab routine and the CAD model of rotor blade is built.

$$
\begin{gathered}
R_{c}=\min \left\{\begin{array}{c}
\left(R_{4}-R_{5 h}\right) \\
L_{a x, r o t}
\end{array}\right. \\
L_{a x, r o t}=1.5\left(R_{5 s}-R_{5 h}\right) \\
R=R_{5 s}+\left(R_{4}-R_{5 s}\right) \xi^{n} \\
\xi=\frac{z-z_{5}}{L_{a x, r o t}-b_{4}}
\end{gathered}
$$

$$
\begin{aligned}
& \theta_{s}(m)=\gamma_{1} m_{s}+\gamma_{2} m_{s}^{3}+\gamma_{3} m_{s}^{4} \\
& \theta_{h}(m)=\gamma_{4} m_{h}+\gamma_{5} m_{h}^{2}+\gamma_{6} m_{h}^{3}
\end{aligned}
$$

$$
\begin{gathered}
\theta_{s}\left(m_{L E}\right)=\tan \beta_{m e t, 4} / R_{4} \\
\theta_{s}\left(m_{T E}\right)=\tan \beta_{m e t, 5 s} / R_{5 s} \\
\theta_{h}\left(m_{L E}\right)=\tan \beta_{m e t, 4} / R_{4} \\
\theta_{h}\left(m_{T E}\right)=\tan \beta_{m e t, 5 h} / R_{5 h}
\end{gathered}
$$

\subsection{CFD Analysis}

Once suitable 3D models of stator and rotor geometries have been generated for the selected candidates, stationary and rotating passages are meshed in ANSYS TurboGrid by means of structured hexahedral meshes, imposing $\mathrm{y}^{+}$values $\approx 1$ on the blade surface and $\approx 5$ on endwalls and blade tip. It is worth mentioning that stator and rotor computational domains were enlarged upstream and downstream, respectively, not to force non-physical solutions due to circumferential non-uniformities introduced by blade and vane presence. Finally, to reduce the computational effort, one passage per row was simulated, exploiting the geometrical periodicity. To save computational time and to accurately model the flow physics, fluid properties were described by means of a Look-Up Table (LUT) approach, and a 200x200 size table was generated by means of thermodynamic library.

CFD calculations were then run by means of steady-state RANS model in ANSYS CFX, with k- $\omega$ SST turbulence model. Advection scheme was set to high resolution, which automatically blends between first order and second order, whereas turbulence was set to first order. A mixing-plane approach was implemented for stator rotor interface to account for the difference in rotational speed. Total temperature and pressure were set for the upstream CFD boundary condition, i.e. stator inlet; static pressure was set at rotor outlet, in accordance with values in Tab. 2.

Convergence was monitored both considering RMS of the residuals and selected quantities at component interfaces.

Table 2. Test cases turbine design boundary conditions

\begin{tabular}{ccccccccc}
\hline $\begin{array}{c}\text { Fluid } \\
-\end{array}$ & $\begin{array}{c}\text { p01 } \\
(\mathbf{b a r})\end{array}$ & $\begin{array}{c}\mathbf{T}_{01} \\
(\mathbf{K})\end{array}$ & $\begin{array}{c}\text { p5 } \\
(\mathbf{b a r})\end{array}$ & $\begin{array}{c}\dot{\boldsymbol{m}} \\
(\mathbf{k g} / \mathbf{s})\end{array}$ & $\begin{array}{c}\mathbf{p}_{01} / \mathbf{p 5} \\
(-)\end{array}$ & $\begin{array}{c}\text { Vol exp } \\
(-)\end{array}$ & $\begin{array}{c}\mathbf{P}_{\text {is }} \\
(\mathbf{k W})\end{array}$ & $\begin{array}{c}\boldsymbol{\Delta h}_{\boldsymbol{T S}, \boldsymbol{i s}} \\
(\mathbf{k J} / \mathbf{k g})\end{array}$ \\
\hline $\mathrm{R} 245 \mathrm{fa}$ & 28.00 & 439.15 & 2.162 & 0.4920 & 12.95 & 14 & 26.89 & 54.65 \\
$\mathrm{R} 245 \mathrm{fa}$ & 10.81 & 403.28 & 2.162 & 0.7690 & 5.00 & 5 & 26.89 & 34.97 \\
MM & 18.00 & 579.75 & 1.020 & 0.2460 & 17.65 & 21 & 18.24 & 74.16 \\
\hline
\end{tabular}




\section{Results}

\subsection{Preliminary Design}

The meanline design code was run for the boundary conditions reported in Tab. 2 and for each test case a parametric analysis was performed by running the code for the turbine design variables combination defined by the ranges reported in Tab. 1. The results of the parametric analysis are presented in the forthcoming by means of contour plots of several quantities as function of flow and work coefficients.

Figure 1 depicts the variations of total-to-static efficiency as a function of flow and work coefficients for the three test cases. Interestingly, all the three test cases present high total-tostatic efficiency values, attained in a rather large portion of the design space, with a smooth decrease moving towards the boundary. As expected, the second test case shows the largest achievable efficiency in a very large portion design space, as a consequence of the lowest volumetric expansion ratio $(\approx 5)$. On the contrary, the third test case, being the most challenging from volumetric expansion ratio perspective $(\approx 21)$, exhibits the lowest total-tostatic efficiency; whereas the first one lies in between the two, attaining a volumetric expansion ratio of nearly 14 . Similar considerations can be drawn for total-to-total efficiency contour as well, Fig. 2.

ORC Turbines expand high molecular weight gases under large expansion ratios, these occurrences are likely to prompt the onset of transonic and supersonic flows, making the design more challenging and eventually requiring special care in the design of blade and vane passages. Figure 3 shows the variation of rotor inlet absolute Mach number as a function of flow and work coefficient, with the additional superposition of the locus of maximum totalto-static efficiency. As one can notice, the maximum efficiency curves, spans over a wide variety of Mach number values ranging from low supersonic to high supersonic, depending on the test case. Whilst test case 2 shows Mach numbers in the low-end range which allows using purely convergent stator, the other two test cases feature substantially higher Mach numbers, making mandatory the use of convergent divergent nozzles.

A widely accepted RITs design best practise recommends keeping the rotor inlet Mach number in the relative frame of reference below unit, in order to enhance efficiency and simplify the design. Figure 4 shows the variation of rotor inlet Mach number in the relative frame of reference as a function of flow and work coefficients, again with the superposition of the locus of maximum total-to-static efficiency. As it can be noticed, the onset on transonic inlet relative Mach number is not of any concern, since it is largely possible to select design candidate characterized by maximum efficiency and subsonic inlet relative Mach. Likewise, rotor outlet relative Mach number is well with subsonic range for candidates in the bestefficiency region, Fig. 5.

The optimal rotor inlet flow angle in the relative frame of refence varies considerably depending on the selected reaction degree, i.e. closer to axial for 0.4-0.5 degree of reaction and towards $50-55^{\circ}$ for very low degree of reaction (say $0.15-0.1$ ). Interestingly, the expansion ratio seems to play a key role concerning the positioning of the maximum efficiency curve over the inlet flow angle map. Actually, the higher the expansion ratio, the higher the minimum rotor inlet flow angle which provides maximum efficiency. In other words, increasing the expansion ratio the best iso-efficiency line is shifted towards higher inlet flow angles.

Optimal rotor outlet flow angle in the relative frame of reference is only weakly affected by the work coefficient, whereas it is strongly influenced by the flow coefficient: to fulfil the constraint given by the optimal kinematic ratio, an increase of the flow coefficient leads to a decrease of the absolute value of the rotor relative outlet flow angle. 
Finally, the blade peripheral speed was found not to be an issue, with maximum value in the maximum efficiency region always below $240 \mathrm{~m} / \mathrm{s}$.

Based on the results of the preliminary design, one geometry per test case was selected, avoiding all geometries characterized by rotor inlet blade height lower than $2 \mathrm{~mm}$. Subsequently, 3D vane and blade shapes have been built by means of the method described in the methodology section; particularly: the second test case stator was built in ANSYS BladeGen, whereas first and third test cases have been built by means of the adapted MoC. Figure 6 shows the design velocity triangles computed by meanline design for the three geometries selected.
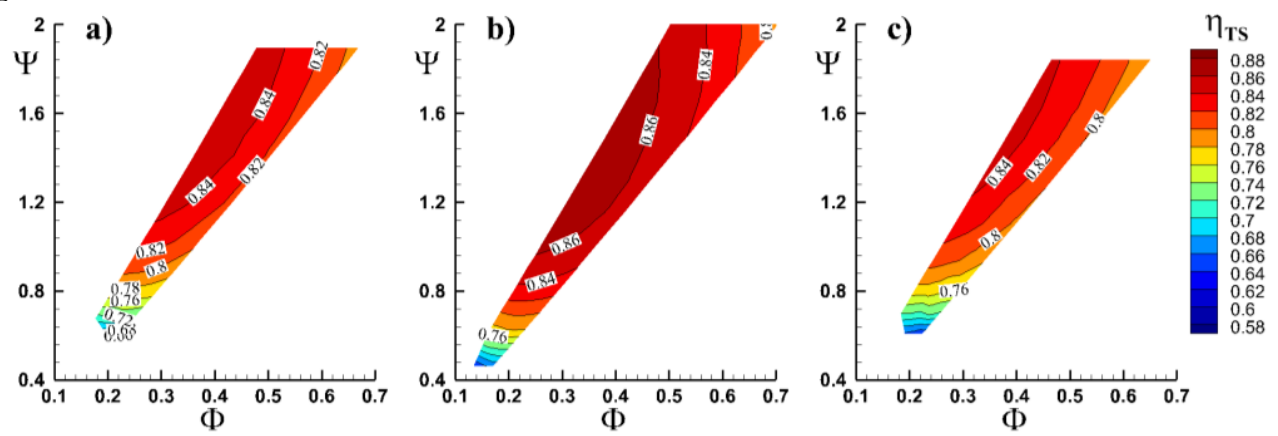

Figure 1. Total-to-Static efficiency contour: (a) Test Case 1; (b) Test Case 2; (c) Test Case 3.
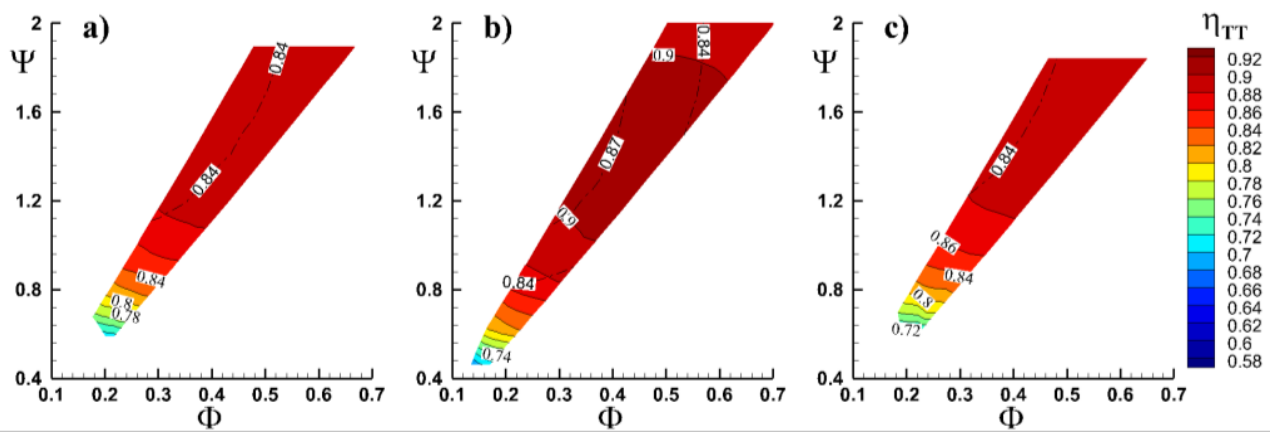

Figure 2. Total-to-Total efficiency contour: (a) Test case 1; (b) Test Case 2; (c) Test Case 3. Locus of maximum total-to-static efficiency with dash dot line
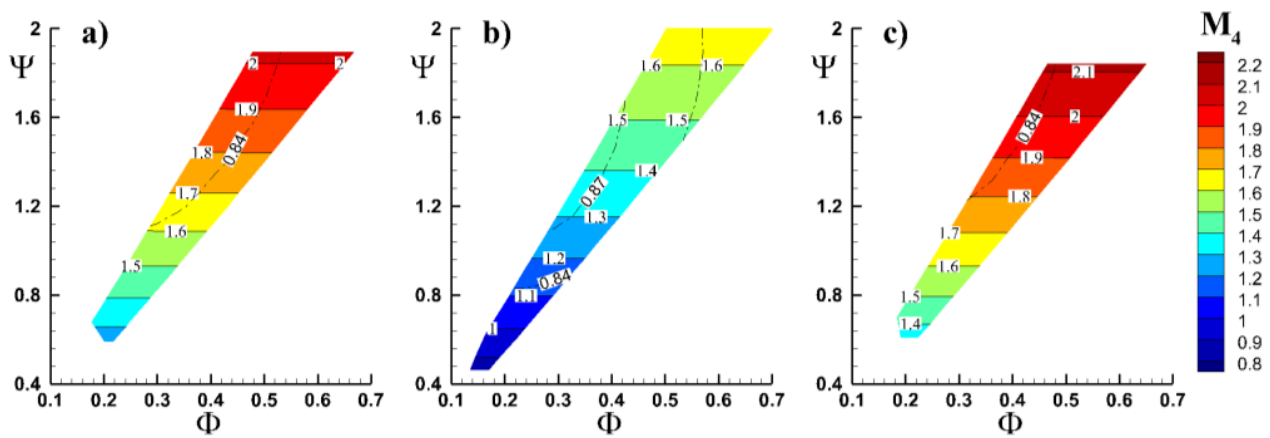

Figure 3. Rotor inlet absolute Mach number contour: (a) Test case 1; (b) Test Case 2; (c) Test Case 3. Locus of maximum total-to-static efficiency with dash dot line 

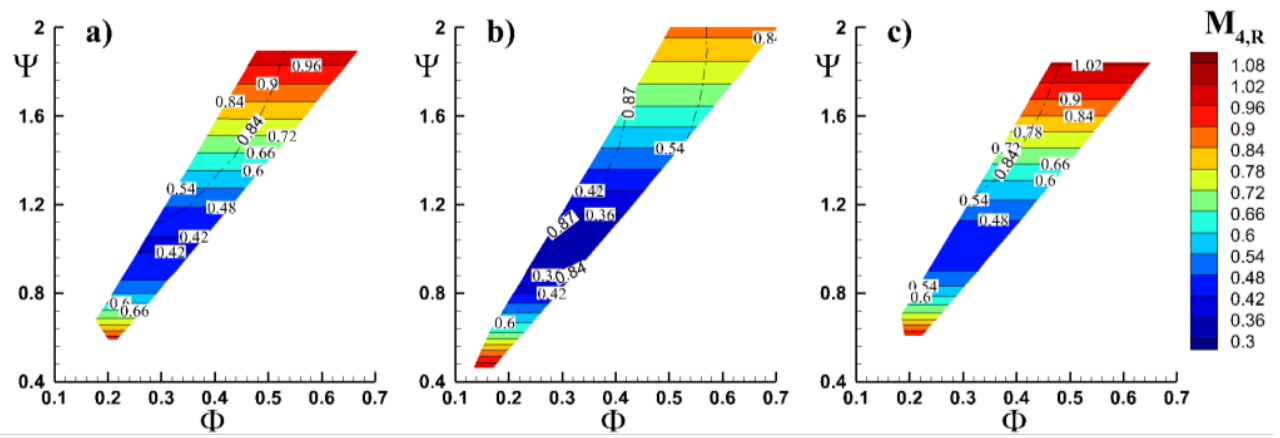

Figure 4. Rotor inlet relative Mach number contour: (a) Test case 1; (b) Test Case 2; (c) Test Case 3. Locus of maximum total-to-static efficiency with dash dot line
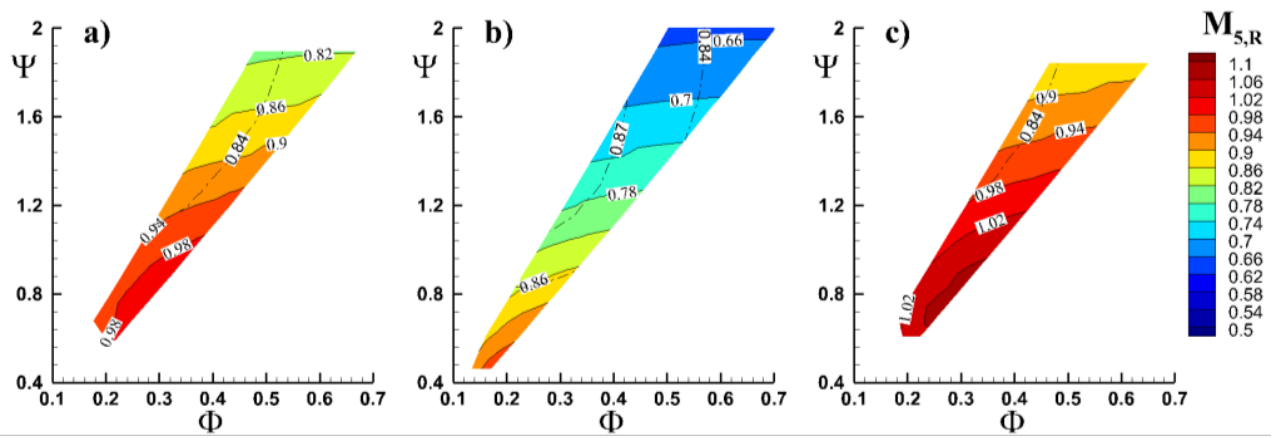

Figure 5. Rotor outlet relative Mach number contour: (a) Test case 1; (b) Test Case 2; (c) Test Case 3. Locus of maximum total-to-static efficiency with dash dot line

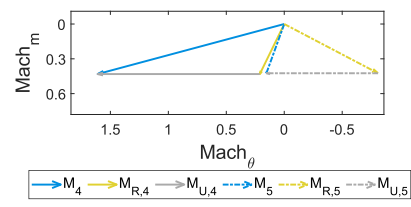

(a)

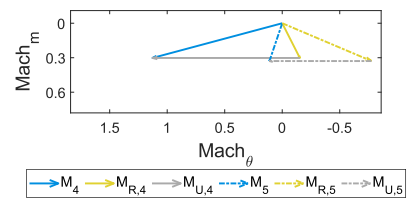

(b)

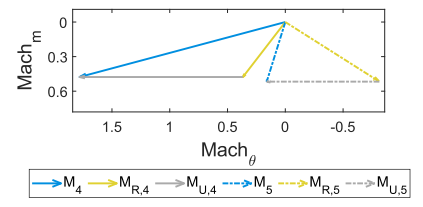

(c)

Figure 6. Selected turbine design velocity triangles: (a) Test Case 1; (b) Test Case 2; (c) Test Case 3.

\subsection{CFD Analysis}

In order to assess the performance of the selected turbines, as well as the accuracy of the loss correlation and mean line model, CFD calculations are required. First of all, a turbine candidate from test case 1, characterized by intermediate levels of Mach number between the three chosen geometries, was selected to carry out a mesh sensitivity analysis. A first mesh was made by setting $\mathrm{y}+$ values $\approx 1$ on the blade surface and $\approx 5$ on endwalls and blade tip. Subsequently, other two meshes were created by refining the first one with a refinement factor $\mathrm{r}=\mathrm{h}_{\text {coarser }} / \mathrm{h}_{\text {finer }}$ greater than 1.3 ; all the characteristic features of the three meshes, say average mesh spacing $\mathrm{h}, \mathrm{y}+$ and refinement, are reported in Tab. 3. Total-to-static efficiency, stator outlet Mach number and rotor outlet relative Mach number, both as absolute values and relative variations were monitored as a function of mesh element number, observing a monotonic convergence behaviour. In view of the results shown in Fig. 7 (in which only the first two quantities are displayed), the accuracy provided by the medium mesh was deemed appropriate for the work and has been used to determine the mesh size for the three selected 
geometries. Considering the different size of the geometries to be analysed, when determining stator and rotor required element number, the target average mesh spacing was correlated to the stator outlet and rotor inlet hydraulic diameters, respectively.

Table 3. Mesh sensitivity analysis information

\begin{tabular}{cccccc}
\hline Mesh & $\mathbf{y}^{+}$blade & $\mathbf{y}^{+}$Endwall & Cells Number $\left(\mathbf{1 0}^{\mathbf{6}}\right)$ & $\mathbf{h}\left(\mathbf{1 0}^{-5}\right) \mathbf{( m )}$ & $\mathbf{r}=\mathbf{h}_{\text {coarser }} / \mathbf{h}_{\text {finer }}$ \\
\hline Coarse & 1 & 5 & 1.21 & 9.32 & - \\
Medium & 1 & 5 & 2.78 & 7.06 & 1.32 \\
Fine & 1 & 5 & 6.18 & 5.41 & 1.30 \\
\hline
\end{tabular}
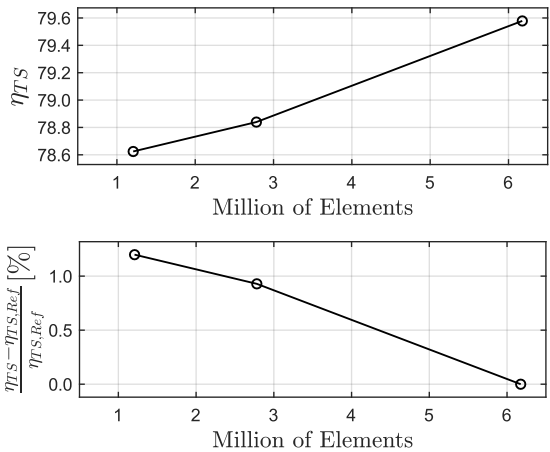

(a)
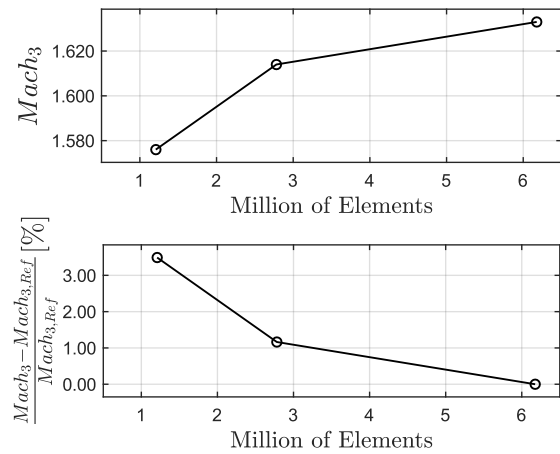

(b)

Figure 7. Mesh sensitivity analysis results as a function of mesh size: (a) total-to-static efficiency; (b) stator outlet Mach number

Table 4 reports a comparison of the efficiency values obtained by the CFD results against the meanline model prediction. Interestingly, a good agreement is found between CFD results and $1 \mathrm{D}$ prediction for the total-to-total efficiency, particularly for the lowest expansion ratio test case, whereas larger discrepancies can be noticed in the total-to-static values. The latter is arguably ascribed to the effect of the tip gap, both on efficiency and rotor outlet flow angle. In fact, despite the meanline model accounts for tip leakage vortex detrimental effect on the efficiency, it does not model its effects on the outlet flow angle.

Table 4. Total-to-total and total-to-static efficiency values: CFD results against meanline model prediction

\begin{tabular}{cccccc}
\hline & \multicolumn{2}{c}{$\eta_{T T}$} & & \multicolumn{2}{c}{$\eta_{T S}$} \\
\cline { 2 - 3 } \cline { 5 - 6 } & 1D & CFD & & 1D & CFD \\
\hline R245fa - Test Case 1 & 88.05 & 83.82 & & 84.46 & 76.40 \\
R245fa - Test Case 2 & 88.29 & 87.69 & & 85.14 & 80.01 \\
MM & 88.60 & 85.81 & & 83.48 & 77.76 \\
\hline
\end{tabular}

The above-mentioned considerations can be confirmed by the outlet velocity triangles obtained in the three cases, i.e. meanline model, mass flow averaged CFD results with and without tip gap, depicted in Fig. 8a. A large discrepancy can be noticed for what concerns the relative outlet velocity vector between mass flow averaged CFD results with tip gap and meanline model, leading in turn the latter to underpredict the outlet absolute velocity and so the leaving kinetic energy. Such discrepancies are, instead, milder in the meanline-CFD results without tip gap comparison. Moreover, quantitative comparison between $1 \mathrm{D}$ results 
and mass flow averaged CFD results with and without rotor tip gap, Tab. 5, shows that a good prediction of rotor relative outlet flow angle is achieved by meanline model in comparison to the no-tip gap case, Tab. 5, whereas larger discrepancies can be noticed in comparison to CFD results with tip gap. As further confirmation, the mass flow averaged relative outlet flow angle change over the blade span is depicted in Fig. $8 \mathrm{~b}$ for the tip gap and no-tip gap cases, showing a large change towards blade tip induced by the tip gap and not accounted in the meanline code.

Finally, the residual discrepancy in the rotor outlet relative flow angle between the meanline prediction and the CFD results without tip gap could be likely ascribed to an underestimation of outlet deviation angle, which in this case was predicted by the Ainley and Mathieson correlation.

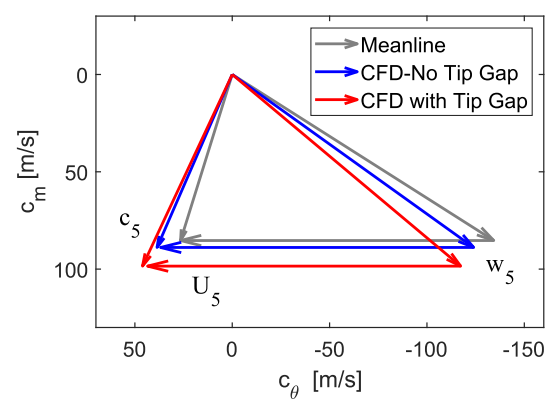

(a)

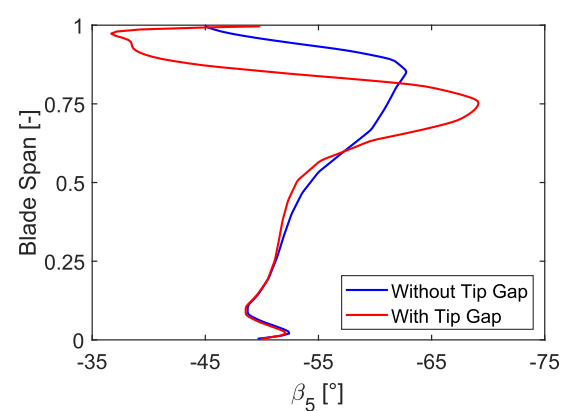

(b)

Figure 8. Outlet velocity triangle comparison: meanline and CFD results with and without tip gap (a); Rotor outlet relative flow angle change over blade span qith and without tip gap (b).

Table 5. CFD results with and without tip gap against meanline model prediction

\begin{tabular}{lcccccc}
\hline & $\eta_{T T}$ & $\eta_{T S}$ & $\beta_{5}$ & $\alpha_{5}$ & $c_{5}$ & $M_{5}$ \\
\hline 1D & 88.60 & 83.48 & -56.25 & 17.82 & 92.93 & 0.563 \\
CFD - No Tip Gap & 88.14 & 82.03 & -54.70 & 23.60 & 100.50 & 0.608 \\
CFD - Tip Gap & 85.81 & 77.76 & -51.00 & 24.57 & 113.30 & 0.6860 \\
\hline
\end{tabular}

Figure 10 shows a comparison of the rotor blade loading for the three test cases, whose 3D geometries are provided in Fig. 9; as one can notice, in all cases a final pressure ripple is present at the terminating part of the suction side. This occurrence is likely to be a consequence of the Aungier's first guess thickness distribution, which produces a rapid flow area change in the final part of the blade passage, inducing an undesired flow acceleration via a Prandtl-Meyer expansion which also has the effect of strengthening the trailing edge shock.

Concerning the first and third test cases, a rather large blade loading can be observed in the front part of the blade. This occurrence is a consequence of the high frontal curvature of the blade, which requires the fluid to undergo a very large turning, leading to a rather large separation on the suction surface in the upper half of the blade span, also noticeable on the pressure distribution, Fig. 10. The second test case blade loading, instead, exhibits rather regular trends at all spans and a light loading, consequence of the modest flow turning.

Figure 11 shows a comparison of the Mach number distributions achieved by the three stator designs and, as it can be noticed, rather regular trends are achieved in all the cases. More specifically, despite a lower expansion ratio, the second case with purely convergent nozzle, Fig. 11b, shows a less regular Mach number distribution, with a slight overexpansion in the frontal part of the suction side, and several waves in the uncovered part of the channel, which might arguably be explained by an excessive curvature of the rear suction side. 
On the other hand, the results achieved by the stators designed by the adapted MoC are particularly interesting. In fact, despite the very high expansion ratio and design Mach number, quite regular distributions can be observed in the contours in Fig. 11 a and c, both in the kernel and in the transition region of the divergent portion of the nozzle, as well as in the uncovered part of the suction side. This occurrence can also be observed in Fig. 12, which displays the stator blade loading achieved by the $\mathrm{MoC}$ design method. A very regular pressure distribution is observed in both cases and only little pressure ripples can be detected in Fig. 12 at the suction side probably caused by impinging shock waves.

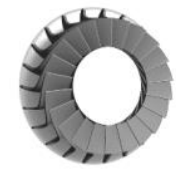

(a)

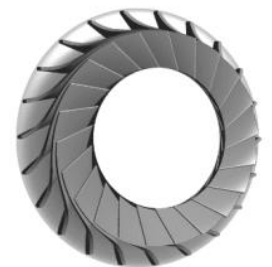

(b)

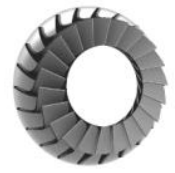

(c)

Figure 9. 3D Rotor Geometries: a) Test Case 1; b) Test Case 2; c) Test Case 3.

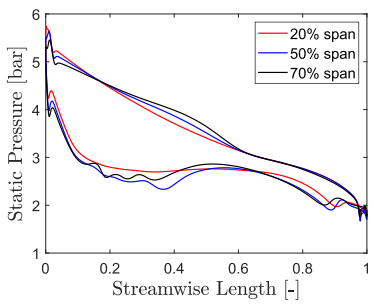

(a)

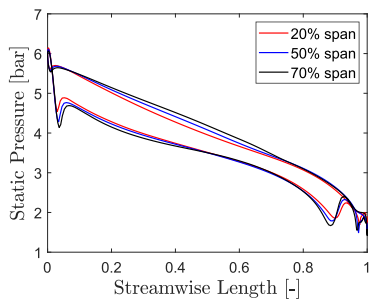

(b)

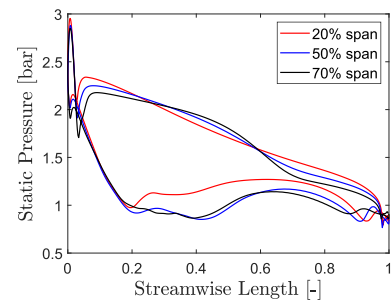

(c)

Figure 10. Rotor blade loading: (a) Test Case 1; (b) Test Case 2; (c) Test Case 3.

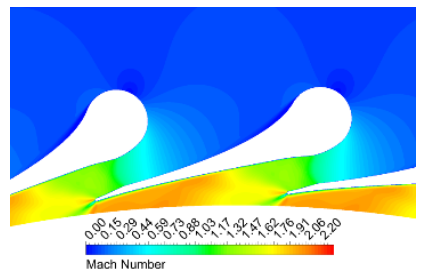

(a)

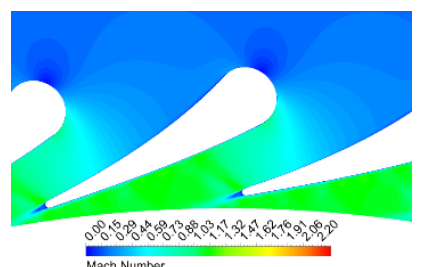

(b)

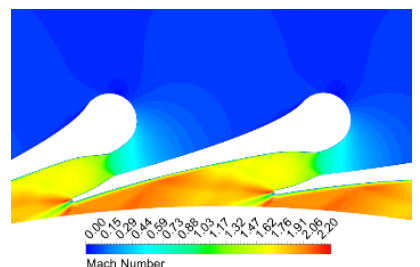

(c)

Figure 11. Stator Mach number contour at 50\% span: (a) Test Case 1; (b) Test Case 2; (c) Test Case 3

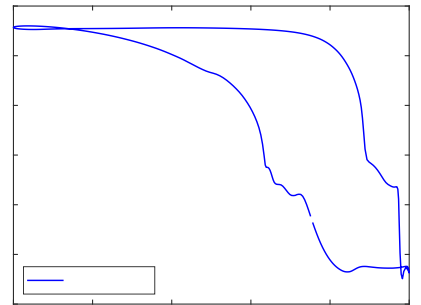

(a)

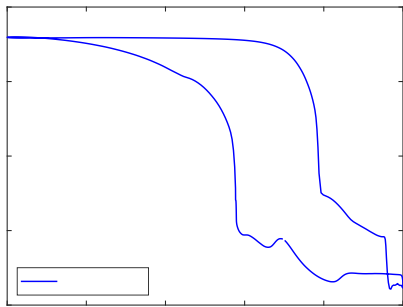

(b)

Figure 12. Stator blade loading of Method of Characteristics design: (a) R245fa; (b) MM. 
Additionally, the effect of the rotational speed on rotor suction side separation was investigated and the results are shown in Fig. 13 which highlights that smoother blade loading are achieved on both pressure side and suction side when increasing the rotational speed. Moreover, increasing the rotational speed by $10 \%$ and $15 \%$ also affected the $\eta_{T S}$ which passed from 76.40 to 77.49 and to 77.0 ; as well as the $\eta_{T T}$, which passed from 83.82 to 86.55 and to 86.73 .

Finally, the effect of blade number variation was investigated for the second test case turbine, by lowering the blade number from 22 to 17 . The resulting blade loading are compared against the original ones at $50 \%$ and $70 \%$ blade span in Fig. 14 and, as expected, the load increased if lowering the blade number. Interestingly, the lower blade number configuration achieved an increase of both $\eta_{T S}$ and $\eta_{T T}$, which passed from 80.01 to 81.54 and from 87.64 to 88.59 , respectively, so suggesting that the blade number correlation might be overestimating the optimal value.

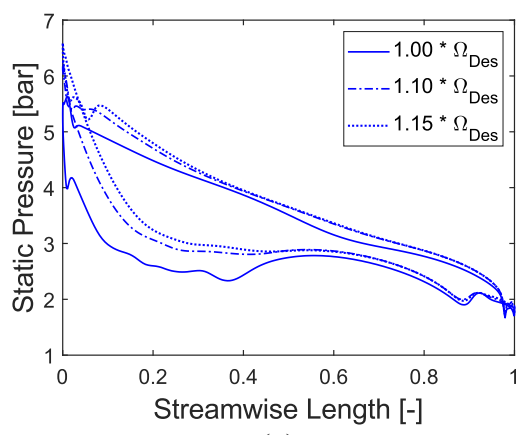

(a)

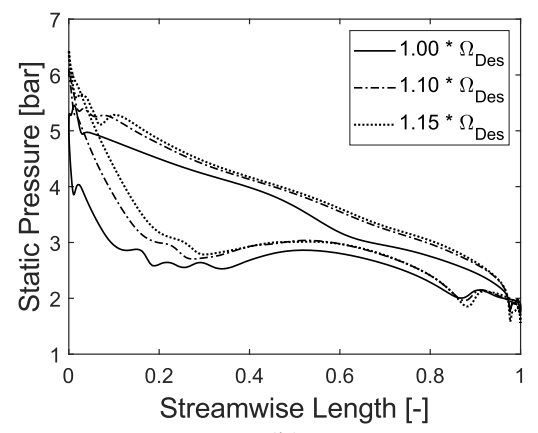

(b)

Figure 13. Effect of rotational speed for Test Case 1: (a) 50\% Span; 70\% Span (b).

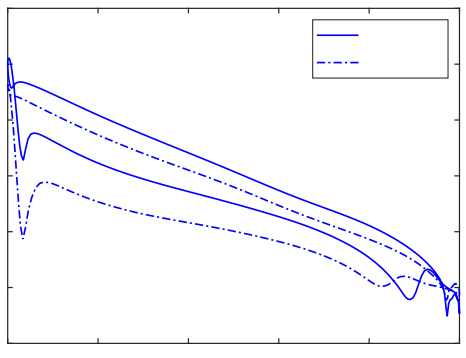

(a)

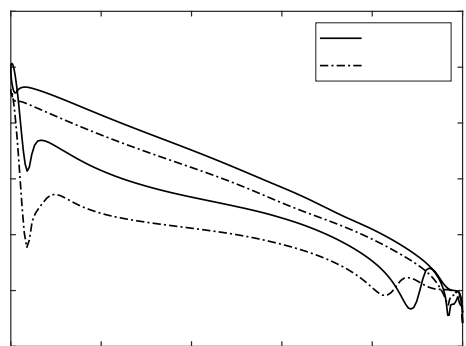

(b)

Figure 14. Blade number effect on blade loading for Test Case 2 : (a) $50 \%$ rotor span; (b) $70 \%$ rotor span.

\section{Conclusion}

In the present work the preliminary design of ORC radial-inflow turbines was carried by means of in-house code accounting for real gas properties for three test cases encompassing differences in fluid, mass flow rate, expansion ratio and volumetric expansion ratio values in the tens of kilowatt scale. The code was used to perform parametric analyses by varying the design parameters and the results were used to build contour maps for several quantities as a function of flow and work coefficients. The code proved to be sensitive to the different operating conditions, showing substantial changes in the optimal efficiency region shape and 
extension, as well as maximum achievable efficiency. The results have been used to investigate the optimal design requirements in terms of stator outlet Mach number, rotor inlet and outlet relative Mach number. While in the lowest expansion ratio case optimal efficiency was found to be achievable by low supersonic stator discharge conditions, for which conventional radial inflow nozzles might still be considered, in the high expansion ratio cases the optimal efficiency was found to require high supersonic stator discharge conditions, imposing the use of less established convergent-divergent radial inflow nozzles, whose geometry might be defined by means of modified Method of Characteristics.

For what concerns rotor relative inlet and outlet Mach numbers, the parametric analyses showed that, despite the very high expansion ratios considered, it is still possible to achieve the maximum predicted efficiency values guaranteeing subsonic rotor inlet conditions and subsonic or weakly supersonic rotor relative discharge conditions.

In the second part of the work most promising turbine candidates were chosen, and 3D geometries were built and analysed by means of CFD steady state calculations. The results showed that the meanline code provides satisfyingly accurate modelling of the total-to-total efficiency, although showing a decrease in the accuracy as the expansion ratio increases. Conversely, total-to-static efficiency prediction was found to be less accurate, because of the effect of tip leakage vortex on rotor outlet average flow angle, which was not accounted in the meanline model.

Furthermore, the first guess thickness distribution from Aungier's first guess method was found to produced undesired Prandtl-Meyer expansion in the terminating part of the rotor, suggesting that it might not be appropriate to high molecular weight fluids, for which sonic and supersonic conditions arise more easily. Overall, the Aungier's first guess method was found to be still applicable to produce a good first guess rotor geometry.

Stator design paradigm was instead observed to be substantially different from conventional radial inflow design law and the adapted MoC-based design method allowed to achieve good first guess geometry, with regular Mach number and blade loading distribution.

The rotational speed increase was observed to have a beneficial effect on rotor suction side separation and rotor blade loading and, in turn on the efficiency level, suggesting that the optimal incidence correlation might produce unreliable results for the application considered. Likewise, a reduction of the blade number, assessed for the low expansion ratio case only, showed that the reduction of the blade number, with respect to the value retrieved from correlation, was possible without compromising the efficiency levels.

Finally, if taking into account that only first guess geometries were considered in this work, the analysis showed that it is possible to design very high expansion ratio radial-inflow machines characterized by good efficiency levels.

As future works, the attempt to bring the modelling of tip leakage vortex effect on flow angle into the meanline model is highly recommended, as well as deeper investigations into the optimal rotor incidence correlation and rotor thickness distribution, for which a linear law from leading edge to trailing edge is recommended.

The parametric analysis, the rotor geometry calculation and the stator design are carried out with MATLAB R2019a. The CFD analysis is carried out in ANSYS Fluid Dynamics.

\section{References}

1. P. Colonna, E. Casati, C. Trapp, T. Mathijssen, J. Larjola, T. Turunen-Saaresti e A. Uusitalo, «Organic Rankine Cycle Power Systems: From the Concept to Current Technology, Applications, and an Outlook to the Future,» Journal of Engineering for Gas Turbines and Power, vol. 137, n. 10, pp. 100801-1-100801-19, (2015) 
2. S. Quoilin, M. Van Den Broek, S. Declaye, P. Dewallef e V. Lemort, «Techno-economic survey of Organic Rankine Cycle (ORC) systems» Renewable and Sustainable Energy Reviews, vol. 22, pp. 168--186, (2013).

3. K. Rahbar, S. Mahmoud, R. K. Al-Dadah, N. Moazami e S. A. Mirhadizadeh, «Review of organic Rankine cycle for small-scale applications» Energy conversion and management, vol. 134, pp. 135--155, (2017).

4. E. Macchi, «Theoretical basis of the Organic Rankine Cycle» in Organic Rankine Cycle (ORC) Power Systems, Woodhead Publishing, pp. 3-24, (2017).

5. V. Mounier, L. E. Olmedo e J. Schiffmann, «Small scale radial inflow turbine performance and pre-design maps for Organic Rankine Cycles» Energy, vol. 143, pp. 1072 - 1084, (2018).

6. L. Da Lio, G. Manente e A. Lazzaretto, «A mean-line model to predict the design efficiency of radial inflow turbines in organic Rankine cycle (ORC) systems» Applied Energy, vol. 205, pp. 187 - 209, (2017).

7. R. H. Aungier, «Aerodynamic Performance Analysis of Radial-Inflow Turbines» in Turbine Aerodynamics: Axial-Flow and Radial-Flow Turbine Design and Analysis, ASME, (2006).

8. R. H. Aungier, «Preliminary Aerodynamic Design of Radial-Inflow Turbine Stages» in Turbine Aerodynamics: Axial-Flow and Radial-Flow Turbine Design and Analysis, ASME, (2006).

9. S. Bahamonde, M. Pini, C. De Servi, A. Rubino e P. Colonna, «Method for the preliminary fluid dynamic design of high-temperature mini-organic rankine cycle turbines,» Journal of Engineering for Gas Turbines and Power, vol. 139, n. 8, p. 082606, (2017).

10. A. Perdichizzi e G. Lozza, «Design criteria and efficiency prediction for radial inflow turbines» in ASME 1987 International Gas Turbine Conference and Exhibition, (1987).

11. D. Fiaschi, G. Manfrida e F. Maraschiello, «Thermo-fluid dynamics preliminary design of turbo-expanders for ORC cycles» Applied Energy, vol. 97, pp. 601 - 608, (2012).

12. C. M. De Servi, M. Burigana, M. Pini e P. Colonna, «Design Method and Performance Prediction for Radial-Inflow Turbines of High-Temperature Mini-Organic Rankine Cycle Power Systems» Journal of Engineering for Gas Turbines and Power, vol. 141, n. 9, (2019).

13. P. Klonowicz, F. Heberle, M. Preißinger e D. Brüggemann, «Significance of loss correlations in performance prediction of small scale, highly loaded turbine stages working in Organic Rankine Cycles» Energy, vol. 72, pp. 322 - 330, (2014).

14. R. Persky e E. Sauret, «Loss models for on and off-design performance of radial inflow turbomachinery» Applied Thermal Engineering, vol. 150, pp. 1066 - 1077, (2019).

15. F. Alshammari, A. Karvountzis-Kontakiotis, A. Pesiridis e P. Giannakakis, «Off-design performance prediction of radial turbines operating with ideal and real working fluids» Energy Conversion and Management, vol. 171, pp. 1430 - 1439, (2018).

16. A. Meroni, M. Robertson, R. Martinez-Botas e F. Haglind, «A methodology for the preliminary design and performance prediction of high-pressure ratio radial-inflow turbines» Energy, vol. 164, pp. 1062 - 1078, (2018).

17. A. Cappiello, R. Tuccillo, M. C. Cameretti e A. Pesyridis, «Axial Flow Turbine Concept for Conventional and e-Turbocharging» in 14th International Conference on Engines \& Vehicles, (2019). 
18. I. H. Bell, J. Wronski, S. Quoilin e V. Lemort, «Pure and Pseudo-pure Fluid Thermophysical Property Evaluation and the Open-Source Thermophysical Property Library CoolProp» Industrial \& Engineering Chemistry Research, vol. 53, n. 6, pp. 2498--2508, (2014).

19. A. P. S. Wheeler e J. Ong, «The Role of Dense Gas Dynamics on Organic Rankine Cycle Turbine Performance» Journal of Engineering for Gas Turbines and Power, vol. 135, n. 10, (2013).

20. M. T. White, C. N. Markides e A. I. Sayma, «Working-Fluid Replacement in Supersonic Organic Rankine Cycle Turbines» Journal of Engineering for Gas Turbines and Power, vol. 140, n. 9, (2018).

21. N. Anand, S. Vitale, M. Pini, G. J. Otero e R. Pecnik, «Design Methodology for Supersonic Radial Vanes Operating in Nonideal Flow Conditions» Journal of Engineering for Gas Turbines and Power, vol. 141, n. 2, (2018).

22. A. Shapiro e G. Edelman, «Method of Characteristics for Two-dimensional Supersonic Flow: Graphical and Numerical Procedures» Massachusetts Institute of Technology, (1947).

23. A. C. Aldo e B. M. Argrow, «Supersonic minimum length nozzle design for dense gases» in The Fifth Annual Thermal and Fluids Analysis Workshop, (1993).

24. D. G. Ainley e G. C. R. Mathieson, «A Method of Performance Estimation for Axial Flow Turbines» British ARC R\&M 2974, (1951). 